\title{
Mean-field modelling of magnetic nanoparticles: The effect of particle size and shape on the Curie temperature
}

\author{
Charles Penny, ${ }^{1}$ Adrian R. Muxworthy, ${ }^{1}$ and Karl Fabian ${ }^{2}$ \\ ${ }^{1}$ Natural Magnetism Group, Department of Earth Science and Engineering, Imperial College London, London, United Kingdom \\ ${ }^{2}$ Geological Survey of Norway, Leiv Eirikssons vei 39, 7491 Trondheim, Norway
}

(Received 5 October 2018; revised manuscript received 26 April 2019; published xxxxxx)

\begin{abstract}
A Heisenberg mean-field model is used to study the effect of size and shape on the Curie temperature of magnetic nanoparticles. Simple cubic, body-centered cubic, and magnetite nanoparticles are modelled as spheres, cubes, and needlelike particles. The Curie temperatures of particles of different shape, but with the same crystal structure and smallest dimension $d$, are found to differ. The range in the value of the Curie temperature between particles of different shape, $\Delta T_{C}$, is found to be $\sim 20 \%$ of the bulk value of $T_{C}$ in particles where $d<10$ atoms. As particle size increases, the value of $\Delta T_{C}$ reduces rapidly and becomes negligible above a threshold size. This threshold size differs between systems and is controlled predominantly by crystal structure. All systems were fit to the finite-size scaling equation, with values of the scaling exponent $v$ found to lie between 0.46 and 0.55 , in good agreement with the expected value of $v=0.5$. No trend in the value of $v$ due to shape was found.
\end{abstract}

DOI: 10.1103/PhysRevB.00.004400

\section{INTRODUCTION}

Interest in magnetic nanoparticles has grown rapidly over the past two decades across a wide range of scientific disciplines: applications in magnetic hyperthermia treatment [1], improved contrast agents for MRI imaging [2], and heatassisted magnetic recording [3] are all actively being pursued. In the environment, magnetic nanoparticles have been proposed as the source of magnetic anomalies over oil fields [4], and their presence in the human brain due to inhalation of anthropogenic pollution has recently been linked to Alzheimer's disease and other neurodegenerative diseases [5]. Despite this interest there are still several fundamental unanswered questions surrounding the magnetic behavior of nanoparticles and other nanoscale structures. Many of these questions arise from a combination of interacting phenomena, making it no longer possible to simply use the bulk parameters to describe the magnetic nanoparticles' behavior.

In bulk magnetic systems, the spin correlation length diverges at the Curie temperature $T_{C}$, but in nanoscale systems the growth of the correlation length is limited by the smallest dimension of the system such that it causes a reduction in $T_{C}$. This obeys a finite-size scaling relationship [6],

$$
\frac{T_{C}(\infty)-T_{C}(d)}{T_{C}(\infty)}=\left(\frac{d_{0}}{d}\right)^{\frac{1}{v}}
$$

where $T_{C}(\infty)$ is the bulk Curie temperature, $d_{0}$ is a characteristic length scale of the system, $v$ is the correlation length scaling exponent, and $d$ is the smallest length scale of the system. Recent developments in preparation techniques have lead to several new experimental investigations of finite-sized scaling in magnetic nanoparticles. However, the results have been varied in contrast with the good agreement between theory and experiment found in studies of thin films $[7,8]$. Studies of hematite and magnetite nanoparticles have found values for $v$ in the range of $0.6-0.8[9,10]$, close to the expected value of 0.7043 [11] for the 3D Heisenberg model. A value of $v=1.06$ was determined from work conducted on Ni nanoparticles [12], with line dislocations near the surface of the nanoparticles suggested as the cause of this discrepancy.

Monte Carlo modelling of nanoscale systems has failed to clarify the situation, with values of $v$ derived from finite-size scaling also failing to agree with the accepted value of the correlation length scaling exponent. An Ising Monte Carlo simulation of maghemite nanoparticles suggested a value of $v=0.49$ from finite sized scaling [13], in clear disagreement with the 3D Ising value of 0.6417 calculated from consideration of thermodynamic derivatives [14]. Simulations of $\mathrm{L}_{0}$-FePt using a classical-spin Heisenberg model determined a value of $v=1.06$ [15], again disagreeing with the generally accepted value. Longrange ordering was suggested as a possible source of this disagreement.

Here we use an atomistic mean-field model based on an approach previously used for analyzing complex magnetic structures [16] and apply it to nanoscale systems. The model was applied to a number of crystal structures; simple synthetic systems with uniform spin and exchange energies and a model of magnetite $\left(\mathrm{Fe}_{3} \mathrm{O}_{4}\right)$. The effect of shape on the Curie temperature of magnetic nanoparticles was studied and finite-size scaling in these systems considered.

\section{MEAN-FIELD MODELLING OF MAGNETIC NANOPARTICLES}

The mean-field approximation is a well understood method for analyzing the behavior of magnetic systems, having been successfully applied across the spectrum of magnetic models from the Ising model to spin glasses $[17,18]$. The generalized mean-field equations for a system of interacting Heisenberg 
$117 \quad i=j$

118 For $j \in N_{i}$

$$
\begin{aligned}
\frac{\partial f_{i}}{\partial m_{j}}= & \frac{2 J_{i j} S_{j}}{k_{B} T}\left[\left(\frac{1}{2 S_{i}}\right)^{2} \frac{1}{\sinh ^{2}\left(\frac{1}{2 S_{i}} \xi_{i}\right)}\right. \\
& \left.-\left(\frac{2 S_{i}+1}{2 S_{i}}\right)^{2} \frac{1}{\sinh ^{2}\left(\frac{2 S_{i}+1}{2 S_{i}} \xi_{i}\right)}\right],
\end{aligned}
$$

119 where

$$
\xi_{i}=\frac{\sum_{j \in N_{i}} 2 J_{i j}\left\langle S_{j}\right\rangle+g \mu_{B} h}{k_{B} T} .
$$

${ }_{120}$ Otherwise for $i \neq j$ and $j \notin N_{i}$,

$$
\frac{\partial f_{i}}{\partial m_{j}}=0
$$

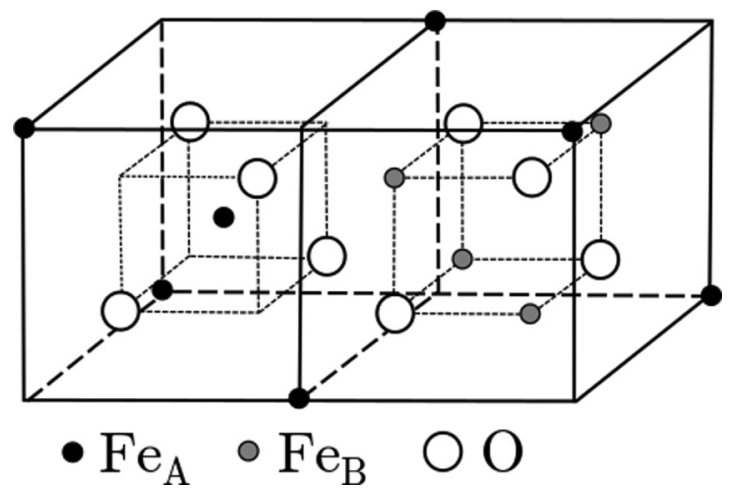

FIG. 1. Structure of magnetite showing two octants of the unit cell. The octants are arranged in a chess board pattern to form the unit cell.

\section{A. Crystal structures and particle shapes}

Particles were modelled using simple cubic, body-centered cubic, and inverse spinel (magnetite) crystal structures. For each crystal structure, five different shapes were studied: cubes, spheres, and three elongated needlelike shapes with aspect ratios of 2:1:1, 5:1:1, and 10:1:1. Each shape was modelled over a range of sizes (see Table I). Spherical particles were approximated on the crystal lattice by including an atom if it lay within $\frac{d}{2}$ of the center of the particle.

Simple cubic (sc) and body-centred cubic (bcc) particles were modelled with an isotropic spin of $S=2$ applied to each atom, and a ferromagnetic exchange energy of $J=3.5 k_{B} \approx$ $0.3 \mathrm{meV}$ between each nearest neighbor.

We also considered magnetite $\left(\mathrm{Fe}_{3} \mathrm{O}_{4}\right)$, as it is an important and well understood natural mineral, which forms in the inverse spinel structure (Fig. 1). 32 oxygen $\left(\mathrm{O}^{2-}\right)$ atoms form a face centered cubic lattice, with 24 iron atoms occupying tetrahedral (A) and octahedral (B) interstitial sites. Eight $\mathrm{Fe}^{3+}$ atoms occupy the $\mathrm{A}$ sites, while the $16 \mathrm{~B}$ sites are occupied by an equal number of randomly distributed $\mathrm{Fe}^{3+}$ and $\mathrm{Fe}^{2+}$ ions [21]. The magnetic structure of magnetite is ferrimagnetic, with the magnetic moments of the A and B sites aligned in opposing directions. The total theoretical moment of magnetite is $4 \mu_{B}$ per formula weight, in close agreement with the experimentally determined value of $4.1 \mu_{B}$ [22].

For the model of magnetite, spins of $S_{A}=2.5$ and $S_{B}=$ 2.25 were assigned to the $\mathrm{A}$ and $\mathrm{B}$ sites. Nearest-neighbor exchange energies of $J_{A A}=-1.56 \mathrm{meV}, J_{A B}=-2.38 \mathrm{meV}$, and $J_{B B}=0.26 \mathrm{meV}$ were used as the most complete set of experimental estimations [23].

\section{B. Determining the Curie temperature}

Previous studies determined the Curie temperature by linearizing the Brillouin function in Eq. (2) and then solving the arising matrix equation for the case of a singular matrix [16]. This approach rapidly became too expensive here given the large number of unique sites even in small particles and so an alternative approach was used.

The solution of the mean-field equations gives the magnetization of each atom in the particle for a given temperature $T$. Below $T_{C}$, this gives a nonzero value of magnetization for each 
TABLE I. Range of smallest length scales, $d$, for all particles modelled in terms of number of atoms. Not all particle diameters in the range were calculated. For inverse spinel systems, particle sizes are also listed in $\mathrm{nm}$ for the case of magnetite.

\begin{tabular}{lccccc}
\hline \hline & & & & \multicolumn{2}{c}{ Needle } \\
\cline { 3 - 6 } & Cube & Sphere & $2: 1: 1$ & $5: 1: 1$ & $2-24$ \\
Simple cubic & $2-45$ & $3-50$ & $2-40$ & $3-37$ & $2-19$ \\
Body-centered cubic & $3-67$ & $5-67$ & $3-41$ & $3-29$ & $8-46$ \\
Inverse spinel & $8-104$ & $8-120$ & $8-76$ & $0.84-5.88 \mathrm{~nm}$ & $0.84-4.62 \mathrm{~nm}$ \\
\hline
\end{tabular}

site; for $T \geqslant T_{C}$, magnetization at each site is zero. It is therefore possible to determine $T_{C}$ by a bisection algorithm. Two initial temperatures were chosen, $T_{L}=1 \mathrm{~K}$ and $T_{H}>T_{C}(\infty)$. The magnitude of the average magnetization of the particle, $\left|\left\langle m_{i}\right\rangle\right|$, at the midpoint between these two temperatures,

$$
T_{M}=\frac{T_{L}+T_{H}}{2},
$$

was calculated. If at this temperature $\left|\left\langle m_{i}\right\rangle\right|$ was found to be greater than a threshold value $\epsilon_{m}=0.0001$, then $T_{M}$ was assumed to be below the Curie temperature, and $T_{L}$ was replaced by $T_{M}$. For $\left|\left\langle m_{i}\right\rangle\right|<\epsilon_{m}, T_{H}$ was replaced by $T_{M}$. This process was iterated, until $T_{H}-T_{L}<0.01 \mathrm{~K}$. The Curie temperature of the particle was then taken as

$$
T_{C}=\frac{T_{L_{f}}+T_{H_{f}}}{2},
$$

where $T_{L_{f}}$ and $T_{H_{f}}$ are the final values of $T_{L}$ and $T_{H}$, respectively, and the error in $T_{C}$ is

$$
\epsilon_{\mathrm{err}}=\frac{T_{L_{f}}-T_{H_{f}}}{2} .
$$

Excellent agreement in the value of $T_{C}$ between the two methods, typically better than $0.02 \mathrm{~K}$, was found in small simple cubic systems.

\section{RESULTS AND DISCUSSION}

\section{A. Properties of nanoparticles}

Normalized magnetization curves, $m(T)$, were calculated in steps of $\Delta T=0.1 \mathrm{~K}$ for bcc and sc particles, and $\Delta T=$ $1 \mathrm{~K}$ for magnetite particles. A sharp Curie-temperature phase transition can be seen for all particles, with the Curie temperature decreasing with decreasing particle size (Fig. 2). In real systems of this size, identifying the Curie temperature is more complex; the superparamagnetic nature of many magnetic nanoparticles requires measurements to be made in the presence of an external field, which destroys the secondorder phase transition, while samples will inevitably contain a distribution of grain sizes and morphologies [24].

The very smallest bcc (not shown) and the magnetite particles have more 'linear' m-T curves than larger particles of the same crystal structure. Their behavior is strongly influenced by atoms in the corner of the cubes, which have only one or two nearest neighbors.

Magnetization varies spatially throughout the nanoparticles at all temperatures (Fig. 3). At low temperatures, a core-shell-like structure is seen, in which a core of atoms behaves in a bulklike manner, surrounded by a shell of atoms exhibiting reduced magnetization. As the temperature increases, this boundary softens, and there is a more gradual change in magnetization throughout the particle. This behavior has previously been observed [25].

\section{B. Effect of varying particle shape}

The Curie temperature of small magnetic nanoparticles is affected by the shape of the particle (Fig. 4). Spherical particles have the lowest Curie temperature for a given size, $d$, with cubic, 2:1:1, 5:1:1, and 10:1:1 particles having successively higher values of $T_{C}$.

It should be noted that the number of atoms in a 10:1:1 particle of size $d$ is an order of magnitude higher than a spherical or cubic particle of the same size. If $T_{C}$ of a 10:1:1 particle is compared to that of a spherical particle containing the same number of atoms (thus larger $d$ ), the sphere has the highest Curie temperature. This is due to the more compact shape of a sphere, which leads to both fewer atoms on the surface and a greater average distance from the surface for atoms inside the sphere.

The normalized difference in the Curie temperature between spherical and 10:1:1 particles is defined as

$$
\Delta T_{C}(d)=\frac{T_{C_{10: 1: 1}}(d)-T_{C_{\mathrm{sph}}}(d)}{T_{C}(\infty)}
$$

and is used as a measure of the strength of the influence of shape on $T_{C}$. In the smallest particles, $\Delta T_{C}$ is found to be

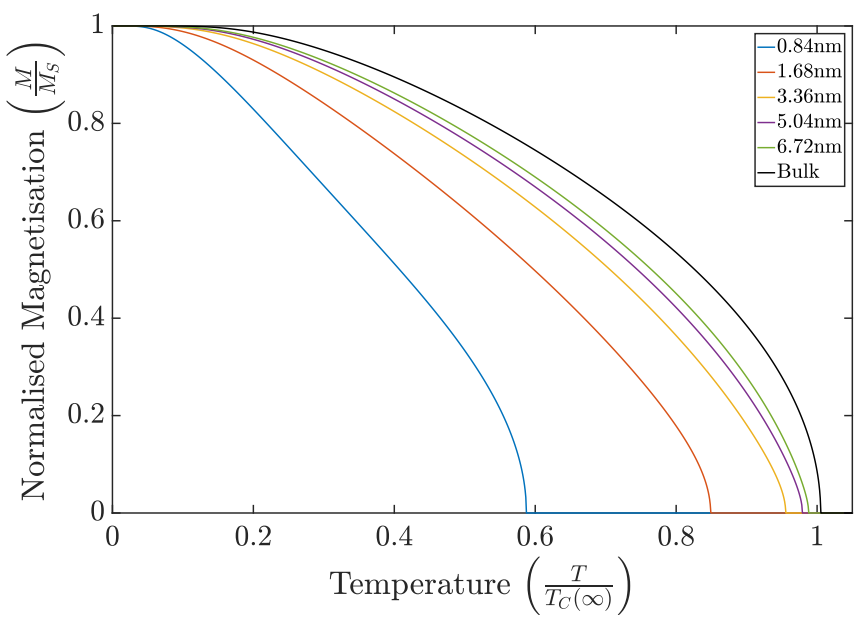

FIG. 2. Magnetization curves of cubic magnetite particles of a number of different sizes. The bulk mean-field magnetization curve is included for reference. Particle sizes are given in $\mathrm{nm}$.

(1)




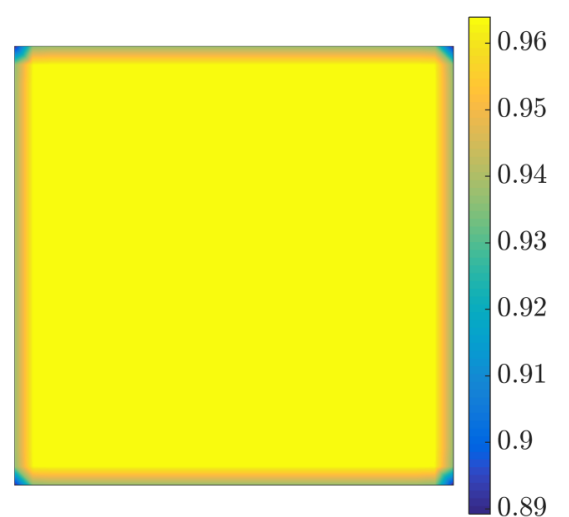

(a)

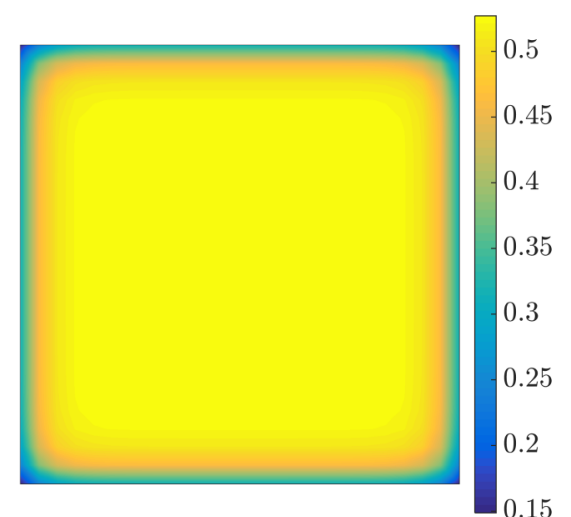

(b)

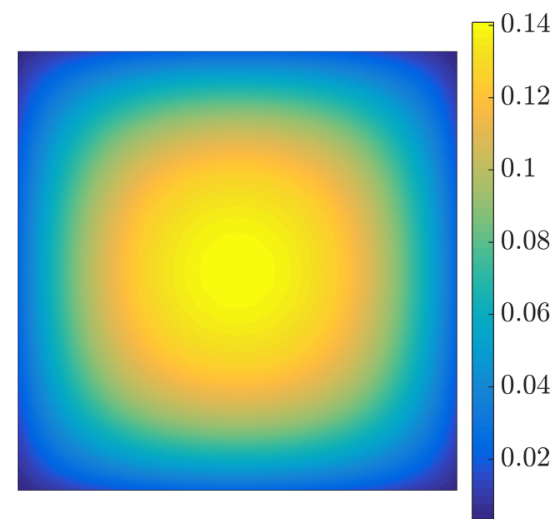

(c)

FIG. 3. Variation of magnetization in a cube of $25 \times 25 \times 25$ atoms arranged in a simple cubic lattice. A slice through the middle of the particle at $z=13$ is shown at three different normalized temperatures: (a) $t=0.36$, (b) $t=0.87$, (c) $t=0.99$, where $t=\frac{T}{T_{C}(d)}$.

$15-25 \%$ of $T_{C}(\infty)$ before it falls off rapidly as particle size increases [Fig. $5(\mathrm{a})]$. The size at which $\Delta T_{C}$ become negligible (taken as $\Delta T_{C}<0.02$ ) varies between crystal structures. In simple cubic and bcc particles this occurs as a size of 10 and 20 atoms, respectively. For magnetite, our results suggest that shape is only an important factor in particles smaller than $5 \mathrm{~nm}(\approx 50$ atoms $)$ in size.

The sensitivity of $\Delta T_{C}$ with respect to the relative magnitudes of exchange energy was tested in magnetite [Fig. 5(c)]. Two additional sets of calculations were made, one using estimations of exchange energies from ab initio calculations [26] and another using uniform values of $J=3.5 k_{B}$. The latter choice creates an artificial ferromagnetic system, with the value of $J$ selected to match the other systems modelled in this work. For all particle sizes, except for the smallest size $d=0.8 \mathrm{~nm}$, the value of $\Delta T_{C}$ does not change appreciably as exchange energies differ. When $d=0.8 \mathrm{~nm}$, a small difference in the value of $\Delta T_{C}$ of 0.05 was seen between the ferromagnetic and two ferrimagnetic systems. This suggests that the relative magnitudes of exchange energies have little bearing on the value of $\Delta T_{C}$ and that the size below which

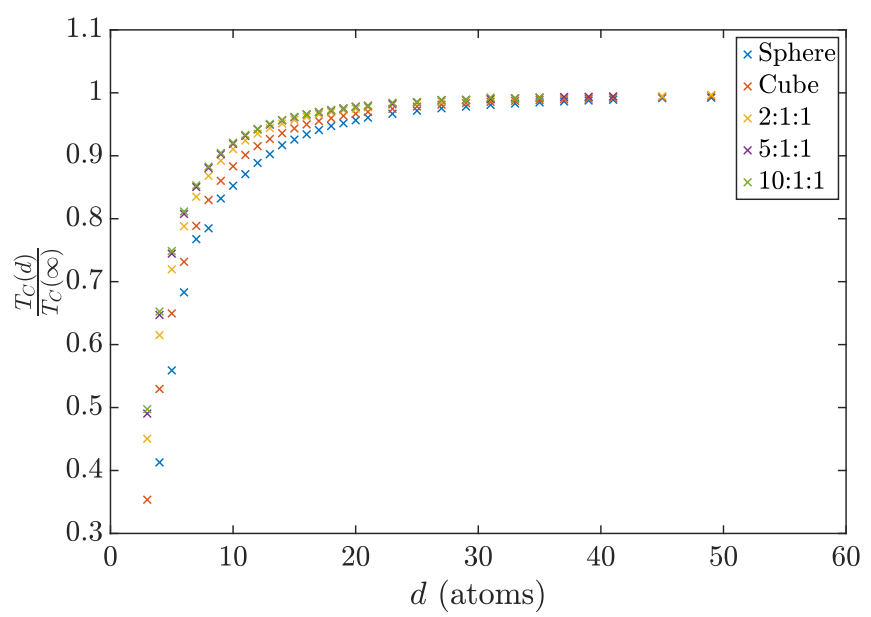

FIG. 4. Normalized Curie temperature of bcc nanoparticles. Data for five shapes is shown: spherical, cubic, 2:1:1, 5:1:1, and 10:1:1 particles. shape begins to have an impact on the Curie temperature is controlled predominantly by crystal structure.

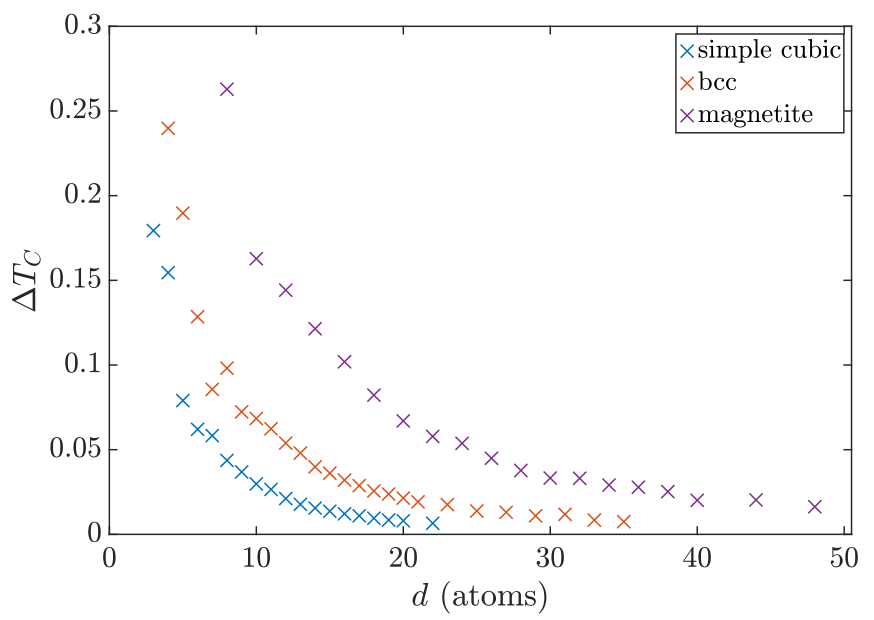

(a)

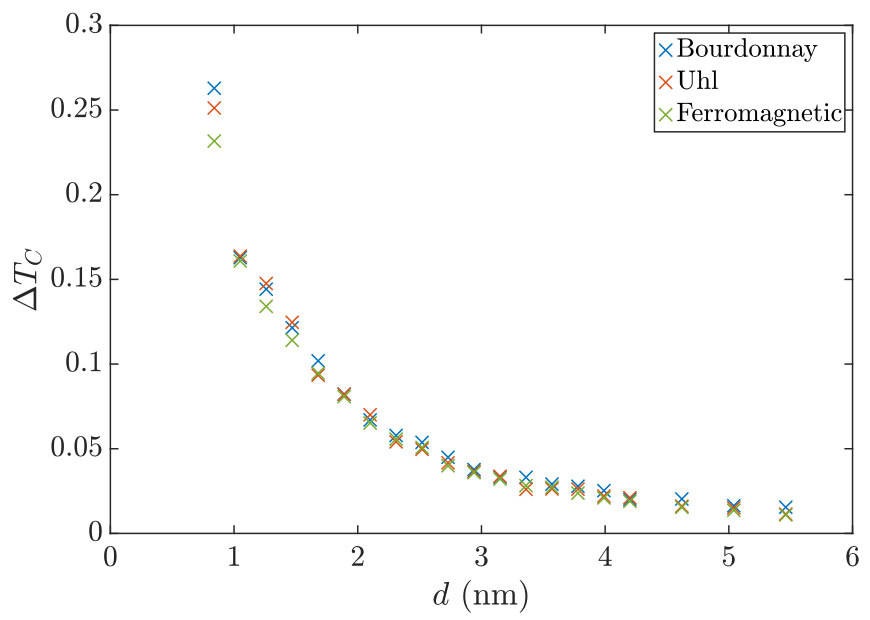

(b)

FIG. 5. $\Delta T_{C}$ as a function of $d$. (a) Illustrates the effect of variation due to crystal structure. (b) Illustrates the effect of changes to exchange energy in magnetite between experiment [23], ab initio modelling [26], and an artificial ferromagnetic system. 


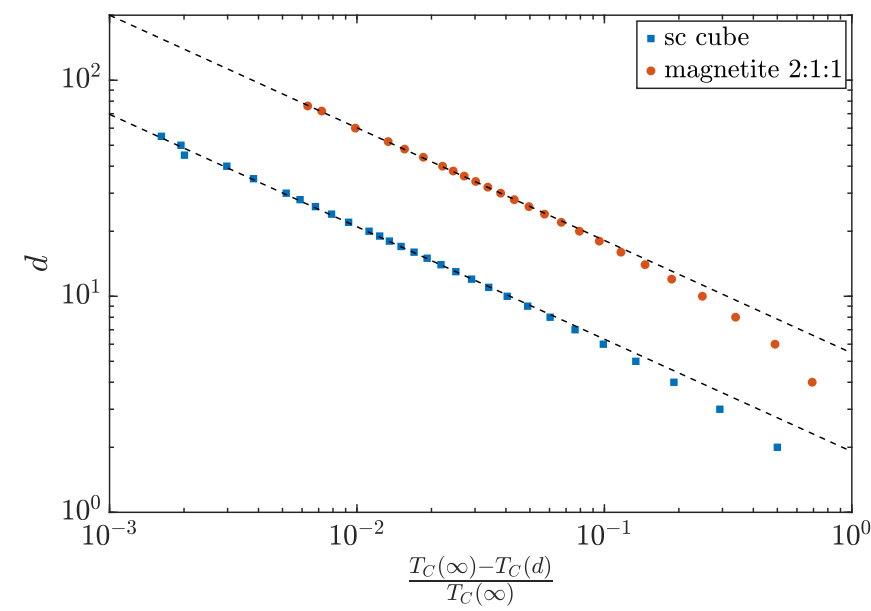

FIG. 6. Example fits to determine the value of the scaling exponent $v$ for cubic sc and magnetite 2:1:1 particles. The dashed lines show the best fits to Eq. (1) where the error in $v$ is minimized.

\section{Finite-size scaling}

Values for the scaling exponent $v$ were calculated by nonlinear least squares regression to Eq. (1) using the LevenbergMarquardt algorithm [27]. The error in $v$ was taken as the $95 \%$ confidence interval of the best fit value. Particles of different materials and shapes were considered separately. Fitting was undertaken initially on all sizes of particles from a particular system and then successively removing the smallest particle from the fit. The final value of $v$ was taken as the one with the smallest error, in order to account for any deviation from scaling behavior seen at small sizes.

The chosen fits describe much of the data well, with deviation away from scaling behavior for diameters smaller than $d \approx 10$ atoms for simple cubic systems, $d \approx 15$ atoms for bcc systems, and $d \approx 20$ atoms for magnetite systems (examples of fitting shown in Fig. 6). Values of $v$ are close to the analytical mean-field result of $v=0.5$ [28] in all cases, lying in the range $0.46-0.55$ (Fig. 7). No trend in the value

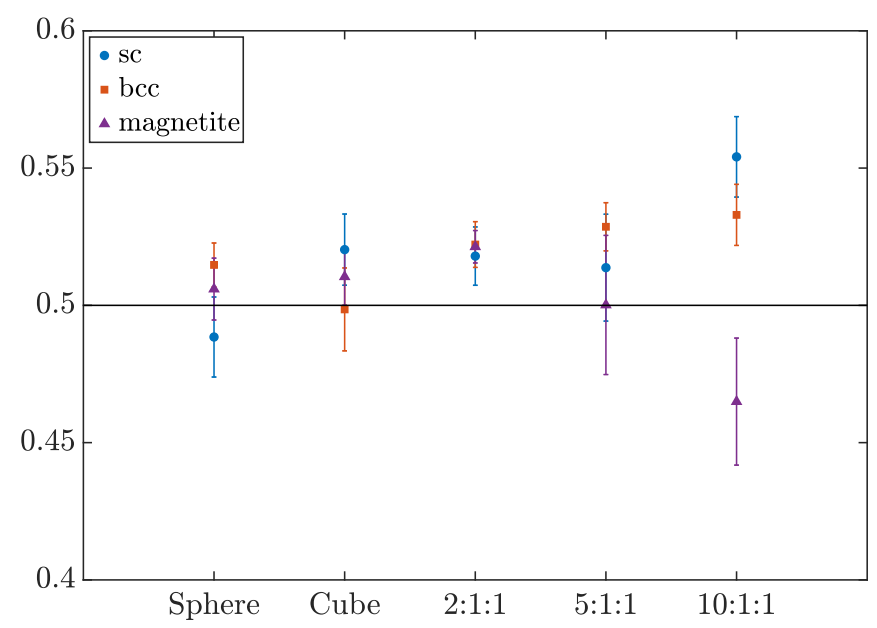

FIG. 7. Calculated values of $v$ for simple cubic, bcc, and magnetite systems. Errors donate $95 \%$ confidence interval of the fit. Solid line highlights the analytical mean-field value of $v=0.5$.

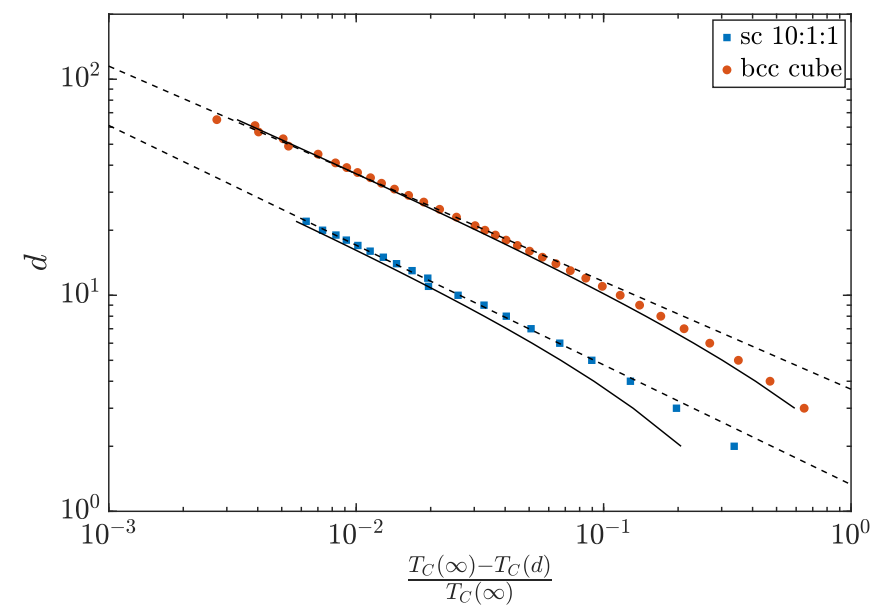

FIG. 8. Comparison of the finite-size scaling law (1), and the modified scaling law (12) in cubic bcc and simple cubic 10:1:1 particles. The solid lines show the analytical results from (12). The dashed lines show the best fit to Eq. (1) where the error in $v$ is minimized.

of the scaling exponent with respect to particle shape can be seen. This consistency of results close to the accepted value of the scaling exponent contrasts with the range of values of $v$ found in other studies $[9,10,12,15]$.

An analytical expression for the Curie temperature of some types of nanoparticle has been derived from Ginzberg-Landau (G-L) theory [25]. This approach has been applied to cubic and long needle particles of simple cubic, body centered cubic, and face centered cubic structure. A modified scaling law is predicted in which

$$
\frac{T_{C}(\infty)-T_{C}(d)}{T_{C}(\infty)}=\kappa\left(\frac{\pi}{d+2}\right)^{2},
$$

where $\kappa$ depends upon the shape and crystal structure of the particle. For a simple cubic structure, $\kappa=\frac{1}{2}$ for a cubic particle and $\kappa=\frac{1}{3}$ for a long needle. For a body centered cubic structure, $\kappa=\frac{3}{2}$ for a cubic particle and $\kappa=1$ for a long needle.

The difference in Curie temperature between 5:1:1 and 10:1:1 particles in the mean-field model is very small (Fig. 4), suggesting that the 10:1:1 particle is a good approximation to a long needle. We can therefore directly compare the analytical result above with the results from the mean-field model. The G-L theory clearly captures the deviation away from finite-size scaling seen in the mean-field approach at very small length scales (Fig. 8). However, the quantitative agreement between the two models varies. For cubic bcc particles, the two models agree very closely, but for simple cubic 10:1:1 particles a larger difference in predictions can be seen; G-L theory predicts a value of $T_{C}$ approximately half of the value of mean-field theory for the smallest systems.

The qualitative accuracy of the modified scaling law was tested by fitting the mean-field data to a law of the general form of (12),

$$
\frac{T_{C}(\infty)-T_{C}(d)}{T_{C}(\infty)}=\left(\frac{d_{0}}{d+2}\right)^{\frac{1}{v}} .
$$




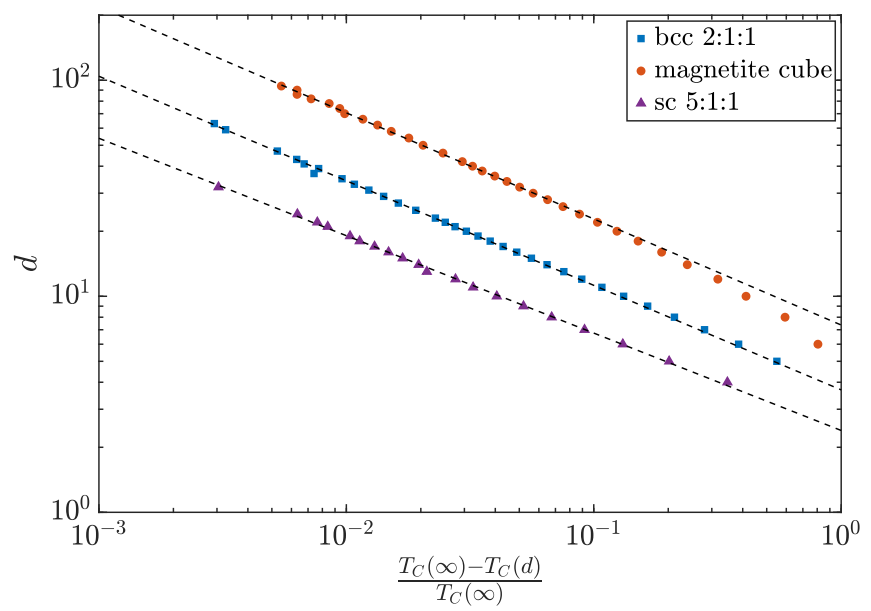

FIG. 9. Example fits to the general modified scaling law (13) for bcc 2:1:1, cubic magnetite, and sc 5:1:1 particles. The dashed lines show the best fits to Eq. (13) where all particles are included in the fit.
In contrast to the fitting procedure for finding $v$ used earlier, only a single fit of all data points was made in order to test (13) across all length scales. The results for simple cubic and bcc particles show that the general form of the modified law describes the scaling behavior at small length scales well (Fig. 9). However, for magnetite particles, Eq. (13) does not describe the scaling behavior at small length scales, with deviation away from this law clearly seen. We suggest this is due to the complex crystal structure of magnetite which involves interactions over many layers of the unit cell. This is in contrast to the simpler sc, bcc, and fcc ordering, which only interact with neighbors in adjacent layers, for which Eq. (12) is derived.

\section{Coordination number}

In the bulk mean-field Heisenberg model the Curie temperature, $T_{C}(\infty)$, is described to a good linear approximation by

$$
T_{C}(\infty)=\frac{(S+1)}{3 S} \frac{2 z J S^{2}}{k_{B}},
$$

where $S$ is the isotropic spin, $z$ is the number of nearest neighbors, and $J$ is the isotropic exchange energy. In the bulk case, the Curie temperature is linearly dependent on the number of nearest neighbors in the system, assuming all other variables are held constant. In view of the relationship between $T_{C}$ and $z$ in (14), it may seem reasonable to assume that $T_{C}(d)$ can be described to a good approximation by the average coordination number $\langle z\rangle$,

$$
T_{C}(d)=\frac{(S+1)}{3 S} \frac{2\langle z\rangle J S^{2}}{k_{B}} .
$$

By considering the left hand side of the scaling Eq. (1), and substituting in Eqs. (14) and (15), an expression for scaling as a function of average coordination number is then obtained,

$$
t=\frac{T_{C}(\infty)-T_{C}(d)}{T_{C}(\infty)}=1-\frac{\langle z\rangle}{z} .
$$

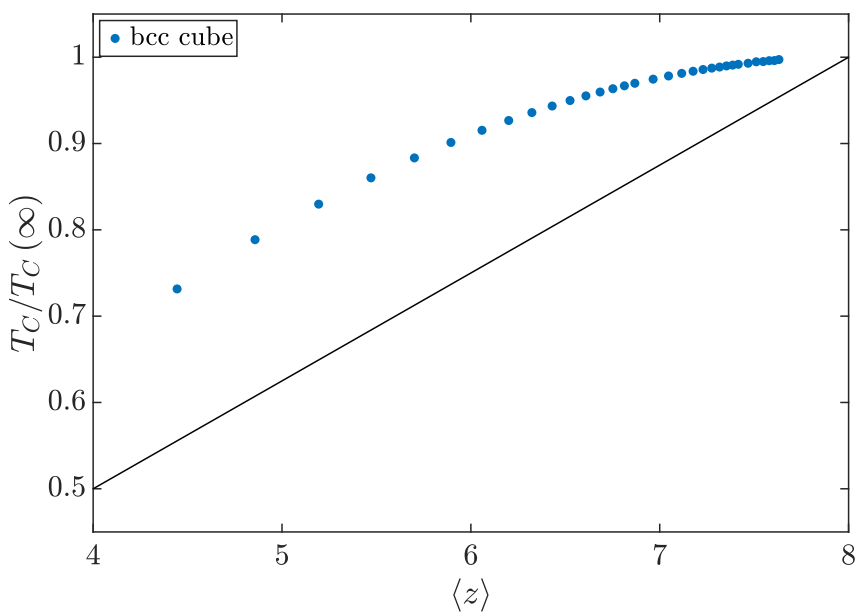

FIG. 10. Plot of reduced Curie temperature against average coordination number for bcc nanoparticles. The solid line shows the linear relationship suggested in (15). No agreement between this relationship and the numerical results can be seen.

Analytic expressions for $\langle z\rangle$ in cubic sc and bcc particles can be found and are given by

$$
\left\langle z_{\mathrm{sc}}\right\rangle=6-\frac{6}{d}
$$

and

$$
\left\langle z_{\mathrm{bcc}}\right\rangle=\frac{4\left(2 d^{3}-6 d^{2}+6 d+8\right)}{d\left(d^{2}+3\right)} .
$$

By further substituting in the expressions for $\left\langle z_{\mathrm{sc}}\right\rangle$ and $\left\langle z_{\mathrm{bcc}}\right\rangle \quad{ }_{325}$ into (16) expressions for $t_{\mathrm{sc}}$ and $t_{\mathrm{bcc}}$ in terms of $d$ are obtained. For a simple cubic system, where $z=6$,

$$
\frac{T_{C}(\infty)-T_{C}(d)}{T_{C}(\infty)}=1-\left(\frac{6-\frac{6}{d}}{6}\right)=\frac{1}{d},
$$

which is a power law of the form in Eq. (1) where $v=1$ and $d_{0}=1$. For a bcc system, where $z=8$,

$$
\frac{T_{C}(\infty)-T_{C}(d)}{T_{C}(\infty)}=1-\frac{\left(2-\frac{6}{d}+\frac{6}{d^{2}}+\frac{8}{d^{3}}\right)}{2+\frac{6}{d^{2}}} .
$$

The $d^{-2}$ and $d^{-3}$ terms quickly become small compared to the $d^{-1}$ term, and so,

$$
\frac{T_{C}(\infty)-T_{C}(d)}{T_{C}(\infty)} \rightarrow 1-\left(\frac{2-\frac{6}{d}}{2}\right)=\frac{3}{d},
$$

which again recovers a power law where $v=1$ (and $d_{0}=3$ ). This result is in clear contrast to the mean-field value of $v=0.5$ and shows that the assumption made in (15) does not hold in the mean-field approximation (also shown in Fig. 10). A previous study of magnetic nanoparticles using the free-energy variational principle found that the expression in (16) was true to a first approximation in that model [29]. The value for the scaling exponent obtained by the variational method for both bcc and fcc lattices was found to be
322 
$v=1.0001 \pm 0.0001$, in agreement with the result obtained above.

\section{CONCLUSIONS}

This study has used the mean-field approximation applied to finite systems of Heisenberg spins to investigate the particle size and shape dependence on the Curie temperature of magnetic nanoparticles. A numerical model was developed to solve the generalized mean-field equations for a number of particle shapes and crystal structures.

$T_{C}$ was found to vary between different shapes of particle, with spheres, cubes, 2:1:1, 5:1:1, and 10:1:1 particles having successively higher values of Curie temperature for the same smallest dimension. $\Delta T_{C}$, the difference in Curie temperature between 10:1:1 and spherical particles, was found to be 15$25 \%$ of the value of $T_{C}(\infty)$ in particles a few atoms across and rapidly decreased as particle size increased. The size at which $\Delta T_{C}$ became negligible was found to differ between crystal structures. For magnetite this was found to be $5 \mathrm{~nm} . \Delta T_{C}$ was also found to be insensitive to changes in the exchange energy between neighboring atoms, showing that crystal structure is the primary driver of the differences in $T_{C}$ due to shape.

All systems were fit to the finite-size scaling law and a good fit was found in all cases. Very small particles, typically $d<10-20$ atoms, showed deviation away from finite-size scaling behavior. Values of $v$ were found to lie in the range 0.46-0.55, which compare well to the analytical mean-field value of 0.5 . No trend in the value of $v$ with relation to particle shape was found. A modified scaling law, derived from Ginzberg-Landau theory, accounted for the observed deviation from finite-size scaling in simple cubic and bcc particles, but was not successful when applied to magnetite.

\section{APPENDIX: DERIVATION OF MEAN-FIELD EQUATIONS}

The derivation presented here applies the mean-field approximation to finite systems of spins in three spatial dimensions; it is closely related to work conducted previously [16] but restricts the spin orientations and the applied field to lie in one direction. However, the final set of equations here features a factor of $S_{i}$ inside the Brillouin function which is not present in the former work. This discrepancy arises between (A16) and (A20), which is treated as a single step in the previous work, and so a full treatment is given here for clarity.

The Heisenberg Hamiltonian for a system of $N$ magnetic spins considering exchange and external field $h$ is given by [28]

$$
\mathcal{H}=-2 \sum_{\langle i j\rangle} J_{i j} S_{i} S_{j}-g \mu_{B} h \sum_{i=1}^{N} S_{i},
$$

where $S_{i}$ is the spin of site $i, J_{i j}$ is the isotropic exchange energy $\left(J_{i j}=J_{j i}\right)$ between sites $i$ and $j, g \approx 2$ is the gyromagnetic ratio, $\mu_{B}$ is the Bohr magneton, and $h$ is an external field acting on each site. The sum over $\langle i j\rangle$ is over nearest neighbor pairs. The definition of the Heisenberg Hamiltonian here gives a positive value to ferromagnetic and a negative value to antiferromagnetic exchange energies. The sum of the first term is taken over each nearest neighbor pair of sites and may be rewritten as

$$
\mathcal{H}=-\sum_{i=1}^{N} \sum_{j \in N_{i}} J_{i j} S_{i} S_{j}-g \mu_{B} h \sum_{i=1}^{N} S_{i},
$$

where $N_{i}$ is the set of nearest neighbor spins of site $i$.

The temporal fluctuation $\delta_{i}$ of the $i$ th spin is defined as [18]

$$
\delta_{i}=S_{i}-\left\langle S_{i}\right\rangle,
$$

where $\left\langle S_{i}\right\rangle$ is the average value or magnetization of spin $i$. The Hamiltonian in terms of $\delta_{i}$ may be written as

$$
\begin{aligned}
\mathcal{H}= & -\sum_{i=1}^{N} \sum_{j \in N_{i}} J_{i j}\left(\delta_{i} \delta_{j}+\delta_{i}\left\langle S_{j}\right\rangle+\left\langle S_{i}\right\rangle \delta_{j}\right. \\
& \left.+\left\langle S_{i}\right\rangle\left\langle S_{j}\right\rangle\right)-g \mu_{B} h \sum_{i=1}^{N} S_{i} .
\end{aligned}
$$

The mean-field approximation is taken, in which correlations between fluctuations are neglected (i.e., $\delta_{i} \delta_{j}=0$ ) [18]. This gives the mean-field Hamiltonian,

$$
\begin{aligned}
\mathcal{H}_{\mathrm{MF}}= & -\sum_{i=1}^{N} \sum_{j \in N_{i}} J_{i j}\left(\delta_{i}\left\langle S_{j}\right\rangle+\left\langle S_{i}\right\rangle \delta_{j}\right. \\
& \left.+\left\langle S_{i}\right\rangle\left\langle S_{j}\right\rangle\right)-g \mu_{B} h \sum_{i=1}^{N} S_{i} .
\end{aligned}
$$

Substitution of Eq. (A3) and summation over all sites allows simplification to

$$
\mathcal{H}_{\mathrm{MF}}=\sum_{i=1}^{N} \sum_{j \in N_{i}} J_{i j}\left(\left\langle S_{i}\right\rangle\left\langle S_{j}\right\rangle-2 S_{i}\left\langle S_{j}\right\rangle\right)-g \mu_{B} h \sum_{i=1}^{N} S_{i}
$$

In order to find the equilibrium state of the system, the minimum of the free energy must be found. The free energy $F$ is given in terms of the well known equation $F=-k_{B} T \ln Z$ where $Z$ is the partition function. The partition function is given by the expression

where $\left\{S_{i}\right\}$ is the sum over all possible states of the system defined by $\mathcal{H}$. Substitution of (A6) and the quantization of angular momentum yields the following expression for the partition function,

$$
Z=\exp \left(-\sum_{i=1}^{N} \sum_{j \in N_{i}} \frac{J_{i j}\left\langle S_{i}\right\rangle\left\langle S_{j}\right\rangle}{k_{B} T}\right) \prod_{i=1}^{N} \sum_{\sigma=-S_{i}}^{S_{i}} \exp \left(\xi_{i} \sigma\right),
$$

where

$$
Z=\sum_{\left\{S_{i}\right\}} \exp \left(\frac{-\mathcal{H}}{k_{B} T}\right)
$$

$$
\xi_{i}=\frac{\sum_{j \in N_{i}} 2 J_{i j}\left\langle S_{j}\right\rangle+g \mu_{B} h}{k_{B} T} .
$$



${ }_{414}$ for $Z$ is given by

$$
Z=\exp \left(-\sum_{i=1}^{N} \sum_{j \in N_{i}} \frac{J_{i j}\left\langle S_{i}\right\rangle\left\langle S_{j}\right\rangle}{k_{B} T}\right) \prod_{i=1}^{N} \frac{\sinh \left(\frac{2 S_{i}+1}{2} \xi_{i}\right)}{\sinh \left(\frac{1}{2} \xi_{i}\right)} .
$$

415 The free energy can be calculated by direct substitution,

$$
\begin{aligned}
F= & \sum_{i=1}^{N} \sum_{j \in N_{i}} J_{i j}\left\langle S_{i}\right\rangle\left\langle S_{j}\right\rangle \\
& -k_{B} T \sum_{i=1}^{N} \ln \frac{\sinh \left(\frac{2 S_{i}+1}{2} \xi_{i}\right)}{\sinh \left(\frac{1}{2} \xi_{i}\right)}=F_{1}-F_{2} .
\end{aligned}
$$

416

417
The equilibrium state of the system occurs when the derivative of free energy with respect to each magnetization, $\frac{\partial F}{\partial\left\langle S_{i}\right\rangle}=$ 0 . The two terms of the free energy are considered separately,

$$
F_{1}=\sum_{i=1}^{N} \sum_{j \in N_{i}} J_{i j}\left\langle S_{i}\right\rangle\left\langle S_{j}\right\rangle
$$

419 and

$$
F_{2}=k_{B} T \sum_{i=1}^{N} \ln \frac{\sinh \left(\frac{2 S_{i}+1}{2} \xi_{i}\right)}{\sinh \left(\frac{1}{2} \xi_{i}\right)} .
$$

The partial derivative of $F_{1}$ yields

$$
\frac{\partial F_{1}}{\partial\left\langle S_{i}\right\rangle}=\sum_{j \in N_{i}} 2 J_{i j}\left\langle S_{j}\right\rangle
$$

where the factor of two arises from the sum over all sites. The case of $F_{2}$ requires a little more manipulation but finally obtains the expression

$$
\begin{aligned}
\frac{\partial F_{2}}{\partial\left\langle S_{i}\right\rangle}=\sum_{j \in N_{i}} 2 J_{i j} & {\left[\frac{2 S_{j}+1}{2} \operatorname{coth}\left(\frac{2 S_{j}+1}{2} \xi_{j}\right)\right.} \\
& \left.-\frac{1}{2} \operatorname{coth}\left(\frac{1}{2} \xi_{j}\right)\right],
\end{aligned}
$$

which is related to the Brillouin function by

$$
\frac{\partial F_{2}}{\partial\left\langle S_{i}\right\rangle}=\sum_{j \in N_{i}} 2 J_{i j} S_{j} B_{S_{j}}\left(S_{j} \xi_{j}\right),
$$

or equivalently

$\frac{\partial F_{2}}{\partial\left\langle S_{i}\right\rangle}=\sum_{j \in N_{i}} 2 J_{i j} S_{j} B_{S_{j}}\left(S_{j} \frac{\sum_{k \in N_{j}} 2 J_{j k}\left\langle S_{k}\right\rangle+g \mu_{B} h}{k_{B} T}\right)$.

The sum over $j$ is a summation over the nearest neighbors of site $i$, while the sum over $k$ is a sum over the nearest neighbors of site $j$. A minimum of free energy is therefore found when

$$
\frac{\partial F}{\partial\left\langle S_{i}\right\rangle}=\sum_{j \in N_{i}} 2 J_{i j}\left[\left\langle S_{j}\right\rangle-S_{j} B_{S_{j}}\left(S_{j} \xi_{j}\right)\right]=0 .
$$

Each term in the sum must equal zero to prevent solutions featuring the self interaction of spins, and so the solution to (A18) reduces to $N$ coupled equations,

$$
\left\langle S_{i}\right\rangle=S_{i} B_{S_{i}}\left(S_{i} \frac{\sum_{j \in N_{i}} 2 J_{i j}\left\langle S_{j}\right\rangle+g \mu_{B} h}{k_{B} T}\right) .
$$

By defining the site normalized magnetization $m_{i}=\frac{\left\langle S_{i}\right\rangle}{S_{i}}$, the final form of (A19) is

$$
m_{i}=B_{S_{i}}\left(S_{i} \frac{\sum_{j \in N_{i}} 2 J_{i j} S_{j} m_{j}+g \mu_{B} h}{k_{B} T}\right) .
$$

[1] M. Johannsen, B. Thiesen, P. Wust, and A. Jordan, Int. J. Hyperth. 26, 790 (2010).

[2] L. M. Parkes, R. Hodgson, L. T. Lu, L. D. Tung, I. Robinson, D. G. Fernig, and N. T. K. Thanh, Contrast Media Mol. Imaging 3, 150 (2008).

[3] D. Weller, O. Mosendz, G. Parker, S. Pisana, and T. S. Santos, Phys. Status Solidi A 210, 1245 (2013).

[4] R. Abubakar, A. R. Muxworthy, M. A. Sephton, P. Southern, J. S. Watson, A. Fraser, and T. P. Almeida, Mar. Pet. Geol. 68, 509 (2015).

[5] B. A. Maher, I. A. M. Ahmed, V. Karloukovski, D. A. MacLaren, P. G. Foulds, D. Allsop, D. M. A. Mann, R. TorresJardón, and L. Calderon-Garciduenas, Proc. Natl. Acad. Sci. USA 113, 10797 (2016).

[6] M. E. Fisher and A. E. Ferdinand, Phys. Rev. Lett. 19, 169 (1967).

[7] F. Huang, G. J. Mankey, M. T. Kief, and R. F. Willis, J. Appl. Phys. 73, 6760 (1993).

[8] T. Ambrose and C. L. Chien, Phys. Rev. Lett. 76, 1743 (1996).

[9] L. Li, F. Li, J. Wang, and G. M. Zhao, J. Appl. Phys. 116, 174301 (2014).
[10] J. Wang, W. Wu, F. Zhao, and G. M. Zhao, Appl. Phys. Lett. 98, 083107 (2011).

[11] K. Chen, A. M. Ferrenberg, and D. P. Landau, Phys. Rev. B 48, 3249 (1993).

[12] J. Wang, W. Wu, F. Zhao, and G. M. Zhao, Phys. Rev. B 84, 174440 (2011).

[13] Ò. Iglesias and A. Labarta, Phys. Rev. B 63, 184416 (2001).

[14] A. M. Ferrenberg and D. P. Landau, Phys. Rev. B 44, 5081 (1991).

[15] A. Lyberatos, D. Weller, G. J. Parker, and B. C. Stipe, J. Appl. Phys. 112, 113915 (2012).

[16] K. Fabian, V. P. Shcherbakov, S. A. McEnroe, P. Robinson, and B. P. Burton, Geophys. J. Int. 202, 1029 (2015).

[17] M. Mezard, G. Parisi, and M. A. Virasoro, Spin Glass Theory and Beyond (World Scientific, Singapore, 1987).

[18] K. Christensen and N. R. Moloney, Complexity and Criticality (Imperial College Press, London, 2005).

[19] P. J. Jensen, H. Dreyssé, and K. H. Bennemann, Surf. Sci. 269270, 627 (1992).

[20] S. Balay, S. Abhyankar, M. F. Adams, J. Brown, P. Brune, K. Buschelman, L. Dalcin, V. Eijkhout, D. Kaushik, M. G.

$$
\begin{aligned}
& 426 \\
& 427 \\
& 428 \\
& 429
\end{aligned}
$$

24

$$
430
$$

3

34


Knepley, L. C. McInnes, W. D. Gropp, K. Rupp, B. F. Smith, S. Zampini, and H. Zhang, PETSc Users Manual, Tech. Rep. ANL-95/11 Rev 3.7 (Argonne National Laboratory, 2016).

[21] C. G. Shull, E. O. Wollan, and W. C. Koehler, Phys. Rev. 84, 912 (1951).

[22] D. J. Dunlop and Ö. Özdemir, Rock Magnetism: Fundamentals and Frontiers (Cambridge University Press, Cambridge, 1997).

[23] H. Bourdonnay, A. Bousquet, J. P. Cotton, D. Cribier, B. Farnoux, R. Feuillatre, B. Hennion, J. Jacquier, G. Jannink, R. Kahn, D. Mons, J. Mons, G. Parette, G. Perpy, L. Rouleau, and J. P. Trotin, J. Phys. Colloq. 32, 1182 (1971).
[24] K. Fabian, V. P. Shcherbakov, and S. A. McEnroe, Geochem., Geophys., Geosyst. 14, 947 (2013).

[25] V. P. Shcherbakov, K. Fabian, N. K. Sycheva, and S. A. McEnroe, Geophys. J. Int. 191, 954 (2012).

[26] M. Uhl and B. Siberchicot, J. Phys.: Condens. Matter 7, 4227 (1995).

[27] K. Levenberg, Quart. Appl. Math. 2, 164 (1944).

[28] H. E. Stanley, Introduction to Phase Transitions and Critical Phenomena (Oxford University Press, Oxford, 1987).

[29] E. A. Velásquez, J. Mazo-Zuluaga, J. Restrepo, and Ò. Iglesias, Phys. Rev. B 83, 184432 (2011). 